Bull. Austral. Math. Soc.

47D25, 46н20

VOL. 52 (1995) [327-329]

\title{
A NOTE ON AMENABLE ALGEBRAS OF OPERATORS
}

\author{
P.C. CURTIS, JR. AND R.J. LOY
}

It is shown that an amenable algebra of operators on Hibert space which is generated by its normal elements is necessarily self-adjoint, so it is a $\mathrm{C}^{*}$-algebra.

A Banach algebra $\mathfrak{A}$ is amenable if every derivation $D: \mathfrak{A} \rightarrow X^{*}$ is inner, for every Banach $\mathfrak{A}$-bimodule $X$. Investigation of the implications of this hypothesis on various aspects of the structure of $\mathfrak{A}$ has been under way for some time. In particular there is the motivating result of Johnson $[9]$ that $L^{1}(G)$ is amenable if and only if $G$ is amenable, the profound results of Connes [2] and Haagerup [6] showing that a $\mathrm{C}^{*}$ algebra is amenable if and only if it is nuclear, and the beautiful result of Scheinberg $[14,7]$ that a uniform algebra on a compact space $\Omega$ is amenable if and only if it is $C(\Omega)$. This last result, which amounts to showing that the algebra is self adjoint, is what prompted the present note. Indeed our result can be considered as an extension of Scheinberg's theorem to the non-commutative case.

Note that in general a commutative amenable algebra need not be self-adjoint, [10]. It is shown in [15] that the algebra generated by a compact operator (on Hilbert space) is amenable only if the operator is similar to a normal operator. A different approach, yielding more general results of this nature, is given in [5].

There are many alternative formulations of the notion of amenability, and the power of the idea is well illustrated in the context of homological algebra. For further details see $[1,3,8,12]$. For our purposes we need only the following result $[8$, Proposition VII, 2.30] which is a corollary of a major result [8, VII, 2.29] concerning the splitting of certain admissible short exact sequences of left or right Banach $\mathfrak{A}$-modules where $\mathfrak{A}$ is an amenable Banach algebra. A proof of this latter result which follows directly from the definition of amenability and which avoids a substantial development of homological algebra machinery is contained in [3, Theorem 2.3].

PROPOSITION. Let $X$ be a left module over an amenable algebra $\mathfrak{A}, Y$ a complemented submodule of $X$ which is reflexive as a Banach space. Then $Y$ is an injective $\mathfrak{A}$-module, that is, there exists a projection $P: X \rightarrow Y$ which is an $\mathfrak{A}$-module homomorphism.

Our result is the following; the commutative case gives Scheinberg's theorem.

Received 22nd December, 1994.

Copyright Clearance Centre, Inc. Serial-fee code: 0004-9729/95 $\$ A 2.00+0.00$. 
THEOREM. Let $\mathfrak{A}$ be an amenable algebra of operators on a Hilbert space, and suppose that $\mathfrak{A}$ is generated by its normal elements. Then $\mathfrak{A}$ is in fact self-adjoint, and so is a $C^{*}$-algebra.

Proof: First note that it suffices to prove the result when $\mathfrak{A}$ has an identity. For applying that case to $\mathfrak{A}^{\#}$ gives that $\mathfrak{A}^{\#}$ is a $C^{*}$-algebra, whence so is $\mathfrak{A}$ as it is a closed ideal in $\mathfrak{A}^{\#}$.

Let $\mathfrak{B}$ be the $C^{*}$-algebra generated by $\mathfrak{A}$, and suppose the result fails so that $\mathfrak{A} \neq \mathfrak{B}$. Then by the Hahn-Banach theorem there is a nonzero $\phi \in \mathfrak{B}^{*}$ with $\phi(\mathfrak{A})=0$. Consider the universal representation $\pi$ of $\mathfrak{B}$ on a Hilbert space $\mathcal{H}$. This representation may be lifted to $\mathfrak{B}^{* *}$ and identifies $\mathfrak{B}^{* *}$ with the von Neumann algebra on $\mathcal{H}$ generated by $\pi(\mathfrak{B})$, that is, the weak operator closure of $\pi(\mathfrak{B})$ in $\mathcal{H}$. Furthermore by $[11,10.1 .1]$ the functional $\phi$ extends uniquely to an ultra weakly continuous functional $\bar{\phi}$ on $\mathfrak{A}^{* *}$. Now by [13, Proposition 3.6.4] $\bar{\phi}$ is normal on $\mathfrak{B}^{* *}$; therefore by [13, Proposition 3.6.7] there exists a positive normal functional $\eta$ on $\mathfrak{B}^{* *}$, a partial isometry $V \in \mathfrak{B}^{* *}$, and a vector $\xi \in \mathcal{H}$ such that

$$
\bar{\phi}(S)=\eta(V S)=\left\langle\pi(S) \xi, \pi\left(V^{*}\right) \xi\right\rangle, \quad\left(S \in \mathfrak{B}^{* *}\right)
$$

and

$$
\phi(S)=\left\langle\pi(S) \xi, \pi\left(V^{*}\right) \xi\right\rangle, \quad(S \in \mathfrak{B}) .
$$

In particular, we have

$$
0=\phi(S)=\left\langle\pi(S) \xi, \pi\left(V^{*}\right) \xi\right\rangle, \quad(S \in \mathfrak{A})
$$

so that

$$
\overline{\pi(\mathfrak{A}) \xi} \neq \overline{\pi(\mathfrak{B}) \xi} .
$$

Now both $\overline{\pi(\mathfrak{A}) \xi}, \overline{\pi(\mathfrak{B}) \xi}$ are left $\mathfrak{A}$-modules via $\pi$ in the obvious sense. Being reflexive they are dual modules. Both being closed subspaces of $\mathcal{H}, \overline{\pi(\mathfrak{A}) \xi}$ is complemented in $\overline{\pi(\mathfrak{B}) \xi}$. Thus by the Proposition there is an $\mathfrak{A}$-module projection $P: \overline{\pi(\mathfrak{B}) \xi} \rightarrow \overline{\pi(\mathfrak{A}) \xi}$. But this means that for $S \in \mathfrak{B}, T \in \mathfrak{A}$,

$$
P \pi(T) \pi(S) \xi=\pi(T) P \pi(S) \xi
$$

so that $P \pi(T)=\pi(T) P$.

Now for $T \in \mathfrak{A}$ normal, $\pi(T)$ is normal, so by [4], $P$ also commutes with $\pi(T)^{*}=$ $\pi\left(T^{*}\right)$. Thus $P$ must commute with $\pi(T), T \in \mathfrak{B}$. Since $P$ is the identity on $\overline{\pi(\mathfrak{A}) \xi}$ we have

$$
P \pi(T) \overline{\pi(\mathfrak{A}) \xi}=\pi(T) \overline{\pi(\mathfrak{A}) \xi}
$$

for $T \in \mathfrak{B}$, so that in fact

$$
\pi(T) \overline{\pi(\mathfrak{A}) \xi} \subseteq \overline{\pi(\mathfrak{A}) \xi}
$$


But $\mathfrak{A}$ is unital, so that $\xi \in \overline{\pi(\mathfrak{A}) \xi}$, whence $\pi(T) \xi \in \overline{\pi(\mathfrak{A}) \xi}$ for all $T \in \mathfrak{B}$, so that $\overline{\pi(\mathfrak{B}) \xi}=\overline{\pi(\mathfrak{A}) \xi}$, contrary to our assumption.

\section{REFERENCES}

[1] F.F. Bonsall and J. Duncan, Complete normed algebras (Springer-Verlag, Berlin, Heidelberg, New York, 1973).

[2] A. Connes, 'On the cohomology of operator algebras', J. Funct. Anal. 28 (1978), 248-253.

[3] P.C. Curtis, Jr. and R.J. Loy, 'The structure of amenable Banach algebras', J. London Math. Soc. (2) 40 (1989), 89-104.

[4] B. Fuglede, 'A commutativity theorem for normal operators', Proc. Nat. Acad. Sci. U.S.A. 36 (1950), 35-40.

[5] J. Gifford, 'On amenable operator algebras', (preprint).

[6] U. Haagerup, 'All nuclear C*-algebras are amenable', Invent. Math. 74 (1983), 305-319.

[7] A.Ya. Helemskii, 'Flat Banach modules and amenable agebras', Trans. Moscow Math. Soc. 47 (1985), 199-244.

[8] A.Ya. Helemskii, The homology of Banach and topological algebras (Kluwer, Dordrecht, 1989).

[9] B.E. Johnson, Cohomology in Banach algebras, Memoirs of the American Mathematical Society 127 (American Mathematical Society, Providence, RI, 1972).

[10] B.E. Johnson, 'Two commutative amenable nonsymmetric Banach algebras', J. Austral. Math. Soc. (to appear).

[11] R.V. Kadison and J.R. Ringrose, Fundamentals of the theory of operator algebras, Volume II (Academic Press, New York, 1986).

[12] A.L.T. Paterson, Amenability, Mathematical Surveys and Monographs 29 (Providence, RI, 1988).

[13] G.K. Pederson, $C^{*}$-algebras and their Automorphism Groups, London Mathematical Society Monographs 14 (Academic Press, London, 1979).

[14] M.V. Scheinberg, 'On a characterization of the algebra $C(\Omega)$ in terms of cohomology groups', (in Russian), Uspekhi Mat. Nauk 32 (1977), 203-204.

[15] G.A. Willis, 'When the algebra generated by an operator is amenable', J. Operator Theory (to appear).

Department of Mathematics

University of California

Los Angeles CA 90024

United States of America

e-mail: pccomath.ucla.edu
Department of Mathematics

Australian National University

ACT 0200

Australia

e-mail: rick.loy@maths.anu.edu.au 